\title{
PolyCystic Ovary Syndrome in Monozygotic Twins Concordant for Lipid Profiles in Iranian Reproductive Women
}

\author{
Leila Amini ${ }^{1}$, Fatemeh Oskouie ${ }^{2}$, Babak Behnam $^{3 *}$, Mohammad Reza Sadeghi $^{4^{*}}$
}

\begin{abstract}
Objective: There is evidence for genetics components in PCOS based on familial clustering of cases. We examined the contribution of genetics impact and some lipid/serum profiles to PCOS in a cohort study of Iranian twins.

Materials and Methods: As a prominent predisposing risk factor for PCOS, serum lipid profiles (Triglyceride, Total cholesterol, HDL, LDL, Apo lipoprotein A-I, Apo lipoprotein B and lipoprotein A), were analyzed in 154 subjects of PCOS. The diagnosis of PCOS has been based on the Rotterdam consensus criteria in this study. We also studied 16 identical twin pairs discordant for PCOS to evaluate the effects of environmental risk factors (including lipid indices profiles) for PCOS. Furthermore, we compared these 16 twin pairs with PCOS, in which just 15 (93.75\%) of them was concordant for abnormal lipid profiles. Also the impact of lipid profile in patients with PCOS was confirmed by comparing total 22 identical and non-identical twin pairs, in which 21 out of them (95.45\%) showed abnormal lipid profiles.

Result: PCOS and increased cholesterol level were higher in identical versus non-identical twins with concordance rates of 1.5 and 1.65 (based on the Rotterdam consensus criteria), respectively. Analysis of identical pairs showed weak association between impact of lipid profile and PCOS while in non-identical pairs, the PCOS was associated with an increased environmental risk (lipid profile).

Conclusion: Different lipid indices were affected by environmental and genetic risk factors in PCOS, but the contribution of environmental and genetic factors to the various PCOS-related changes in ovarian structures remains to be established.

Keywords: Lipid Profile, PCO, Rotterdam Criteria, Twins Study
\end{abstract}

\section{Introduction}

Polycystic ovary syndrome (PCOS) is a complex (polygenic) and heterogeneous disorder with no particular pattern of inheritance characterized by hyperandrogenemia, hyperinsulinemia, insulin resistance, and chronic anovulation. PCOS heritability is inferred from studies of the syndrome in different populations while some of variations in the results of genetic studies have been originated from the lack of uniform criteria for diagnosis. However, regardless of used diagnostic criteria, the available genetic studies suggest a familial component. Meanwhile, there is a genetic susceptibility to PCOS with an unknown pathophysiologic and molecular basis.
The high prevalence of PCOS and the wide range of its phenotypic expression can be explained by the interaction of some genes with environmental factors; however its etiology has yet to be clearly elucidated. The impact of environmental factors or genetic predisposition on PCOS and PCOS-associated traits (hyperandrogenisms, hyperinsulinemia, insulin resistance, type 2 diabetes, dyslipidemia, hypertension, obesity, and coronary artery disease) is not exactly clear (1). Clinical genetic studies had been hampered by the lack of consensus regarding the definition of polycystic ovary and PCOS. Main difficulties aroused from the fact that the disorder is clinically expressed in women only during their reproductive years.

Received 4 January 2014, Revised 21 March 2014, Accepted 1 April 2014, Available online 14 April 2014

${ }^{1}$ Department of Midwifery, Nursing and Midwifery School, Iran University of Medical Sciences (IUMS), Tehran, Iran

${ }^{2}$ Nursing Care Research Center and Nursing and Midwifery School, Iran University of Medical Sciences (IUMS), Tehran, Iran

${ }^{3}$ Department of Genetics and Molecular Biology, Faculty of Medicine, Iran University of Medical Sciences (IUMS), Tehran, Iran

${ }^{4}$ Monoclonal Antibody Research Center, Avicenna Research Institute, ACECR, Tehran, Iran

Corresponding Authors:

*These authors contributed equally to this publication

Babak Behnam, Iran University of Medical Sciences, Hemmat and Chamran Highway Cross, Tehran, Iran. Tel: +982186703251, Email: b-behnam@tums.ac.ir

Mohammad Reza Sadeghi, Avicenna Research Institute, Evin, Chamran Expway, Tehran, Iran. Tel: +982122432020, Email: sadeghi@avicenna.ac.ir 
The extent to which familial clustering of PCOS is due to inheritance or shared environmental factors, and can be assessed by comparing concordance rates for the disease in identical versus non-identical twins. Since identical twins are genetically identical while non-identical twins share on average half of their genes, the difference between the concordance rates signifies the contribution of genetic versus environmental factors to the etiology of PCOS. Therefore, we conducted a twin study to measure the extent of PCOS heritability and to identify environmental and genetic factors involved in ethiopathogenesis of PCOS. The purpose of this study was to establish a more reliable concordance rate and baseline heritability that estimate for PCOS in identical versus non-identical twins; and a cohort of intact identical and non-identical female twin pairs as a resource for future studies.

\section{Materials and Methods}

\section{Subjects}

The data of this study, was taken from the subjects utilized and mentioned in our previous survey (2), and was part of Iranian Twin Registry. All of the twins filled a demographic questionnaire and were asked to undergo a medical evaluation for PCOS confirmation. This includes a physical exam, medical history, pelvic ultrasound study, biochemical blood tests, and a clinical evaluation for acne, and hirsutism based on Ferriman-Gallwey Score. The women were also asked for history of irregular menses and zygosity based on their self-reports. After that, all individuals divided in two groups (with and without PCOS) based on Rotterdam criteria. At last, all blood samples stored at $\left(-80^{\circ} \mathrm{C}\right.$ in a freezer for further studies). As a part of a national ongoing twin family study, surveys were mailed to them and their families with an interval of 18 mounts. Data of zygosity were collected from all twins based on the questionnaire data in total 154 (out of the 2000) females participated in the survey. The remaining data set for the univariate analyses of PCOS contained 154 females including 96 identical and 58 non-identical (same sex) twins. Because of our announcement for only femalefemale twins, we had no females from non-identical opposite sex pairs, or non-twin sisters.

\section{Phenotype definition}

PCOS was evaluated based on questions about the annum frequency of menstrual cycles while not using contraception ( $>8,<9,<6,2$ or less), suffering from acne/pimples, and hirsutism (including physical exam based on Ferriman Galway score). Then PCOS was defined as less than nine natural menstrual cycles a year combined with hirsutism or acne. In addition, the survey included some demographic information (date of birth, age at menarche, birth weight, current height and weight, and having children).
Statistical analyses

Modeling of twin data allows discrimination between phenotypic variance due to genetic factors and environmental factors. We assessed the additive genetic effects of contributing gene loci reflecting the dominance and narrow-sense heritability of PCOS via analyzing the concordance rates. The concordance rates were counted and a comparison analysis was done among identical and non-identical twins and between them for the presence of PCOS. Variance caused by shared environmental effects are also assessed and specified in our model.

\section{Results}

Based on the Rotterdam consensus criteria, concordance rate was higher among identical (33.34\%) than nonidentical (20.69\%) twin pairs with the ratios of 1.65 for occurrence of PCOS, and 1.5 for the level of cholesterol, respectively. Characteristics and demographic data of the studied individuals are listed in Table 1. The prevalence for abnormal HDL varied from $43.75 \%$ in identical versus $16.67 \%$ in non-identical twins indicating a concordance risk ratio of 2.625. Although this ratio was significantly less (1.125) for LDL, and the prevalence of the whole lipid profile shows no significant difference between identical and non-identical twins. In total, $67.3 \%$ of the females included in this study have abnormal lipid profile. The percentage of females suffering from PCOS was not significantly different in females with abnormal lipid profile ( $p=0.783$ ). In contrast, the females with abnormal lipid profile showed more PCOS than the females with normal lipid profile $(\mathrm{p}<0.05)$. There were 72 females $(46.75 \%)$ classified as having PCOS. The prevalence was $48(66.7 \%)$ in identical versus $24(33.3 \%)$ in non-identical which seems to be significantly different $(\mathrm{p}<0.05)$. Table 2 shows the tetrachoric correlation values for identical and non-identical twin and sister pairs. Interestingly and significantly, the highest rate of PCOS occurrence has been observed in 1 out of 2 non-identical females, and correlation values are higher in identical twins than non-identical ones suggesting a large genetic influence on all variables. The pattern of correlations for PCOS also suggests additive genetic influences. Considering the HDL values among identical versus non-identical females with PCOS, a significant correlation (> 2.65 folds) has been observed, suggesting genetic dominance or epistasis. To distinguish between genetic and common environmental influences, univariate genetic models were recruited and fitted to the PCOS data (Table 3). Also in the full model, $77.4 \%$ of the variance is explained by genetic factors (additive genetic variance), $5.5 \%$ by shared environment factors (common environmental variance), and $17.1 \%$ by unique environmental factors. The latter results suggested that the roles of shared environmental factors are also significant. The larger statistical power on 
Table 1. Demographic data of identical and non-identical twins with and without PCOS.

\begin{tabular}{ccccccc}
\hline & \multicolumn{3}{c}{ IDENTICAL $(\mathrm{n}=\mathbf{9 3})$} & \multicolumn{3}{c}{ NON-IDENTICAL $(\mathrm{n}=59)$} \\
\cline { 2 - 7 } & $\begin{array}{c}\text { PCOS- } \\
(\mathrm{n}=57)\end{array}$ & $\begin{array}{c}\text { PCOS }+ \\
(\mathrm{n}=36)\end{array}$ & P Value & $\begin{array}{c}\text { PCOS }- \\
(\mathrm{n}=36)\end{array}$ & $\begin{array}{c}\text { PCOS+ } \\
(\mathrm{n}=21)\end{array}$ & P Value \\
\hline Age(years) & $20.11 \pm 5.80$ & $22.11 \pm 5.57$ & 0.10 & $22.75 \pm 6.68$ & $22.19 \pm 8.58$ & 0.78 \\
Menarche age(years) & $12.46 \pm 1.15$ & $12.67 \pm 1.40$ & 0.43 & $12.83 \pm 1.38$ & $12.52 \pm 1.25$ & 0.41 \\
BMI (kg/m $\left./ \mathbf{m}^{2}\right)$ & $23.16 \pm 5.06$ & $24.25 \pm 4.75$ & 0.30 & $23.06 \pm 4.88$ & $21.11 \pm 3.80$ & 0.12 \\
\hline
\end{tabular}

BMI, body mass index

Table 2. A tetrachoric correlation value for PCOS among identical and non-identical twin, and sister pairs is shown. The highest rate of PCOS is observed in 1 out of 2 non-identical females, and correlation values are higher in identical twins than non-identical ones suggesting a large genetic influence on all variables.

\begin{tabular}{cccc} 
& $\begin{array}{c}\text { IDENTICAL } \\
\text { (48 twin pairs) }\end{array}$ & $\begin{array}{c}\text { NON-IDENTICAL } \\
\text { (29 twin pairs) }\end{array}$ & Concordance Rate \\
\hline Concordant PCOS & $16(33.34 \%)$ & $6(20.69 \%)$ & 1.65 (conc. Rate) \\
Discordant PCOS & $16(33.34 \%)$ & $12(41.38 \%)$ & - \\
None PCOS & $16(33.34 \%)$ & $11(37.93 \%)$ & - \\
\hline
\end{tabular}

Table 3. Concordant lipid profile (in Identical twins). Considering the HDL values as the prominent lipid profile among identical versus nonidentical females with PCOS, a significant correlation ( $>2.65$ folds) is observed. To distinguish between genetic and common environmental influences, univariate genetic models were recruited and fitted to the PCOS data (see 3 below panels).

\begin{tabular}{ccccc}
\hline & & Concordant Normal & Concordant Abnormal & Discordant abnormal \\
\hline & LDL & $11 / 16$ & $3 / 16$ & $2 / 16$ \\
Concordant lipid profile & HDL & $3 / 16$ & $7 / 16$ & $6 / 16$ \\
(in Identical twins) & CHOL & $4 / 16$ & $4 / 16$ & $8 / 16$ \\
& TG & $10 / 16$ & $4 / 16$ & $2 / 16$ \\
\hline & LDL & $4 / 6$ & $1 / 6$ & $1 / 6$ \\
Concordant lipid profile & HDL & $1 / 6$ & $1 / 6$ & $4 / 6$ \\
(in Non-Identical twins) & CHOL & $1 / 6$ & $2 / 6$ & $3 / 6$ \\
& TG & $4 / 6$ & $2 / 6$ & 0 \\
\hline Concordance rates of & LDL & $4 / 6$ & 1.12 & - \\
lipid profiles (Identical vs & HDL & $1 / 6$ & 2.625 & - \\
Non-Identical) & CHOL & $1 / 6$ & 0.75 & - \\
\hline
\end{tabular}

familial resemblance than the univariate model, clearly shows the importance of genetic factors to PCOS.

\section{Discussion}

In this study, we announced for female (identical and nonidentical) twins to study the rate of PCOS and other traits associated with it. First, a concordance rate of $33.34 \%$ among identical and $20.69 \%$ among non-identical twin pairs, with the ratio of 1.65 was found. We have also reached 1.5 as a concordance ratio rate for the level of cholesterol among identical compared to non-identical twin pairs in Iran. In a previous twin study of PCOS, there was a concordance rate of $60 \%$ among the sampled nonidentical twin pairs and $74 \%$ among identical twin pairs (3). This difference might be due to different inclusion criteria of Rotterdam as well as a more susceptibility to this complex disease in Iranian individuals in the 2 decades. Hence, assigning a phenotype to prepubertal girls and postmenopausal women is complicated. Moreover, the syndrome is associated with subfertility, so large pedigrees with multiple affected women are hard to be found (4). Besides the controversy about the exact nature of a possible male phenotype (4), it has been suggested that premature balding is more prevalent among male relatives of women with PCOS (5). Nevertheless, the genetic basis of WHO II/PCOS is demonstrated by many studies showing an increased prevalence in individuals who have a first degree relative with the disease (6-8). However, these studies are flawed by aberrant diagnostic criteria and inaccurate diagnostic methods. Despite all shortcomings the existing literature suggests clustering of PCOS in families with a mode of inheritance that is consistent with an autosomal dominant pattern (4).

In the largest available study including both identical and non-identical twins, Jahanfar et al. (1995) found a high degree of discordance among the available twins 
for polycystic ovaries. However, there was considerable concordance among affected twins with respect to biochemical parameters, including fasting insulin levels and androgens. These results indicate that both genetic and environmental factors might be involved in the susceptibility to PCOS. This survey was conducted to estimate the impacts of genetics as well as some environmental factors on PCOS in Iranian population based on updated Rotterdam diagnostic criteria, and some para-clinical parameters, respectively.

In a recent study, no significant difference has been shown between the prevalence of PCOS in women from opposite-sex and same-sex twin pairs, singleton sisters, or spouses; indicating no intrauterine environmental exposure to male fetus, results in PCOS-like traits (9). In complex disorders (polygenic trait) such as PCOS, several genetic and environmental factors may be implicated. Hence the mutation-associated risk is heavily depending on the presence of other genetic or environmental risk factors. Therefore, PCOS might result from the interaction of susceptible and protective genomic variants and environmental factors, during either prenatal or postnatal life (10). As it is often impossible to clinically distinguish between patients who developed the disease due to a specific mutation and those who have a different etiology, recombination between the disease and marker may be falsely inferred. Therefore, the power to unravel the genetics of complex disorders has proven to be low (11).

\section{Conclusion}

As a further study, a genetics investigation to identify the genes with high impact on PCOS occurrence, and interacting with environmental factors is highly recommended.

\section{Ethical issues}

The local ethics committee approved the study.

\section{Conflict of interests}

Authors declare that there is no conflict of interests.

\section{Acknowledgments}

Authors would like to appreciate Avicenna Research Institute (ARI) collaboration with Tehran University of
Medical Sciences (TUMS) and Iran Nursing Care Research (INCR) via recruiting the Bank of Twin Pairs. We also thank Dr. Sara Pajouhanfar for her help in organizing some analyses of the data.

\section{References}

1. Salmi DJ, Zisser HC, Jovanovic L. screening for and treatment of polycystic ovary syndrome in teenagers. Exp Biol Med (Maywood) 2004; 229:369-77.

2. Jahanfar Sh, Maleki H, Mosavi AR. Subclinical eating disorder, polycystic ovary syndrome- is there any connection between these two conditions through leptin- a twin study. Med J Malaysia 2005; 60(4):441-6.

3. Jahanfar S, Eden JA, Warren P, Seppala M, Nguyen TV. A twin study of polycystic ovary syndrome. Fertil Steril 1995;63:478-86.

4. Legro RS, Strauss JF. Molecular progress in infertility: polycystic ovary syndrome. Fertil Steril 2002; 78(3):569-76.

5. Franks S, Gharani N, Waterworth D, Batty S, White $\mathrm{D}$, Williamson $\mathrm{R}$, et al. The genetic basis of polycystic ovary syndrome. Hum Reprod 1997;12:2641-8.

6. Ferriman D, Purdie AW. The inheritance of polycystic ovarian disease and a possible relationship to premature balding. Clin Endocrinol 1979;11: 291.

7. Lunde O, Magnus P, Sandvik L, Hoglo S. Familial clustering in the polycystic ovarian syndrome. Gynecol Obstet Invest 1989;28(1):23-30.

8. Govind A, Obhrai MS, Clayton RN. Polycystic ovaries are inherited as an autosomal dominant trait: analysis of 29 polycystic ovary syndrome and 10 control families. J Clin Endocrinol Metab 1999;84:38-43.

9. Kuijper EA, Vink JM, Lambalk CB, Boomsma DI. Prevalence of polycystic ovary syndrome in women from opposite-sex twin pairs. J Clin Endocrinol Metab 2009;94(6):1987-90.

10. Diamanti-Kandarakis E, Piperi C, Spina J, Argyrakopoulou G, Papanastasiou L, Bergiele A, et al. Polycystic ovary syndrome: the influence of environmental and genetic factors. Hormones (Athens) 2006; 5(1):17-34.

11. Lander ES, Schork NJ. Genetic dissection of complex traits. Science 1994; 265 (5181):2037-48.

Copyright (c) 2014 The Author(s); This is an open-access article distributed under the terms of the Creative Commons Attribution License (http://creativecommons.org/licenses/by/4.0), which permits unrestricted use, distribution, and reproduction in any medium, provided the original work is properly cited. 